\title{
Análisis del Mercado de Oferta y Demanda de Servicios Turísticos en Tacna: Periodo 2003 - 2007
}

RESPONSABLE: CPC Victor Hugo Quispe Cabrera

\begin{abstract}
RESUMEN El objetivo principal del presente trabajo de investigación es diagnosticar la oferta y la demanda de los servicios colaterales del turismo. Del 2004 hasta el año 2007 la tendencia es creciente y el 2008 bajo en 6,9\%, así mismo el índice de pernoctabilidad del turista extranjero en 2008 fue de $78,40 \%$ en relación al 2007 . No existen establecimientos de hospedajes de 4 y 5 estrellas $y$ restaurantes de 4 y 5 tenedores; $y$ las agencias de viajes y turismo no promocionan los sitios turísticos de la ciudad y el departamento de Tacna.
\end{abstract}

En el año 2008 arribaron 793,528 chilenos turistas y visitantes extranjeros, con cédula de identidad, con salvoconducto y/o con pasaporte, quienes ingresaron con diferentes destinos, con la implicancia de una serie de demandas, especialmente servicio de hospedajes, restaurantes y transportes. Asi mismo se observa el ingreso de 727,442 turistas y visitantes por el terminal Collasuyo durante el año 2008 que entró en funcionamiento en los últimos años.
ABSTRACT The main objective of this research work is to diagnose the supply and demand of collateral tourism services. From 2004 to 2007 the trend was growing and 2008 low by $6.9 \%$, also the rate of overnight stays of foreign tourists in 2008 was $78.40 \%$ compared to 2007 , there are not lodging facilities of 4 and 5 star neither restaurants 4 and 5 forks. The travel agents and tourism do not promote the tourist places of the city and department of Tacna.

In 2008 arrived 793.528 Chilean foreign visitors and tourists, with identity cards, with safe conduct and /or passport, who entered with different destinations, with the implication of a lot demands, especially the service of lodging, restaurants and transport. Also we observed the enter of 727.442 tourists and visitors Collasuyo terminal for buses during 2008, It started its operation in recentyears.
INTRODUCCIÓN El turismo es una actividad multisectorial que requiere la concurrencia de diversas áreas productivas -agricultura, construcción, fabricación- y de los sectores públicos para proporcionar los bienes y servicios utilizados por los turistas.

Hoy en día, con el auge del turismo como actividad económica, muchos países ven en ella la mejor manera de incrementar sus divisas, mejorar sus economías y ofrecer servicios a estos curiosos viajeros que lo quieren ver todo. Así encontramos las más diversas formas de practicarlo y que están en constante evolución o transformación. Entre ellas tenemos, el turismo cultural, el solidario, el ecológico, el de aventura, el religioso, el místico, el étnico, el gastronómico, de ocio, el temático, por citar algunas clases de turismo.

En general, con el presente estudio se trata de analizar todos los servicios colaterales de las principales variables inherentes a la industria del turismo, especialmente los referidos a los Establecimientos de Hospedajes, Restaurantes y Agencia de Viajes y Turismo en la región Tacna.

\section{ANTECEDENTES DEL PROBLEMA}

Tacna tiene en el turismo un sector con enorme potencial de desarrollo, ya que cuenta con variados recursos turísticos para atender a todos los segmentos del mercado. Las divisas generadas por el Turismo en el Perú para el año 2005 se estima en 1,378 millones de dólares que representa 227 millones más que el año 2004.

Tacna, como ciudad fronteriza, se ha convertido en el segundo punto de ingreso de turistas al Perú. Asimismo, teniendo como indicador la demanda de Establecimientos de Hospedaje del Turismo Receptivo en 163,245 turistas, un índice de pernoctabilidad de 1.49 y un gasto per cápita promedio diario $\$ 42$ dólares, se ha tenido un ingreso de 10'215,872 dólares, que sumados a los ingresos del Turismo Receptivo se puede afirmar que el Turismo en Tacna representó para el año 2005 un movimiento de 26 millones de dólares, sin estimar las compras que realizan los turistas nacionales en el Sistema ZOFRATACNA de vehículos, artefactos eléctricos, bicicletas y otros similares.

\section{OBJETIVOS DE LAINVESTIGACIÓN}

\section{Objetivo General}

Analizar la oferta y la demanda de los Servicios Turísticos en Tacna: Periodo 2004-2008.

Objetivos Específicos

1. Determinar el número de establecimientos de hospedaje instalados en la ciudad y departamento de Tacna. 
2. Determinar el número restaurantes instalados en la ciudad y departamento de Tacna.

3. Determinar el número agencias de viaje instaladas en la ciudad de Tacna.

4. Analizar las características de las demandas de los servicios turísticos en Tacna.

\section{Población y Muestra}

Población

Las unidades comprendidas fueron las oficinas de la Dirección Regional de Turismo y Comercio Exterior de la ciudad de Tacna.

\section{Muestra}

Dada la magnitud de la población, la investigación tuvo un carácter de observación y revisión documentaria como, así mismo, la evaluación de los archivos, como las estadísticas de ingreso de turistas a la ciudad de Tacna por diferentes lugares y terminales.

\section{FUNDAMENTACIÓN Y JUSTIFICACIÓN DEL ESTUDIO}

\section{Fundamentación}

El turismo es una actividad económica que se viene desarrollando a grandes pasos, convirtiéndose en la industria con tasa de crecimiento más elevada a escala mundial. Este crecimiento ha tenido también impactos directos en otros sectores o ramas como el transporte, la industria, la producción de alimentos, entre otros, acarreando problemas, sobre todo en lo referente a los servicios conexos que se suman de manera importante al turismo, como son, los hoteles, restaurantes y agencias de viajes.

\section{Justificación del Problema}

Un factor importante en la decisión de llevar a cabo esta investigación es el hecho de que en los últimos años la industria turística se considera una buena opción de negocios con una gran posibilidad para lograr éxito como empresario de servicios conexos al turismo (hospedajes, restaurantes y otros) y generar buenas ganancias.

\section{RESULTADOS DE LAINVESTIGACIÓN}

\section{Ofertas o Lugares Turísticos de la ciudad de Tacna:}

Es interesante visitar en la ciudad de Tacna los siguientes lugares: La Catedral, Plaza de Armas ,Pileta Ornamental, Casa de Zela, Teatro Municipal, Museo
Ferroviario, Museo Histórico, Arco Parabólico, Casa Basadre, Alameda Bolognesi, Casa Jurídica, Parque de la Locomotora, Alto de la Alianza y otros.

\section{Oferta de Sitios o Lugares Turísticos del Departamento}

El Balneario Boca del Río es una de las mejores y más concurridas playas ubicada a 53 kilómetros de Tacna.

La Cueva de Toquepala, ubicada a 160 kilómetros de Tacna. Lugar donde se encuentran pinturas rupestres cuya antigüedad data de 7500 a.C.

Poblado de Candarave, ubicado a 164 kilómetros de Tacna. "Lugar de Cóndores" debido a que está localizado al pie del volcán Yucamani.

Laguna de Suches o Huaytire, ubicada a 35 kilómetros de Candarave.

Valle de Locumba, ubicado a 85 kilómetros de Tacna, En la ciudad de Locumba, capital de la provincia, se encuentra el Santuario del Señor de Locumba, también llamado "Señor de los Pies Quemados".

Catarata de Panina: ubicada en Cambaya. Se origina en la cuenca del río Camilaca. Catarata que presenta dos caídas de agua cristalina, con una altura promedio de 30 m. sorteando hermosos paisajes naturales.

Camino Inca Tarata-Santa María: El camino Inca de Tarata a Santa Maria consituye uno de los ramales registrados por el Cápac Ñan.

\section{Oferta de Servicios de Establecimiento de Hospedajes}

En el Cuadro $\mathrm{N}^{\circ} 01$ se observa la estructura de la oferta de los establecimientos de hospedaje existentes en la ciudad de Tacna, se aprecia en 2008 un ligero incremento de $2,7 \%$, en hoteles, en hostales el 12,6\% y en no categorizados en $11,3 \%$.(ver Cuadro $\mathrm{N}^{\circ} 01$ ).

\section{Cuadro $\mathrm{N}^{\circ}$ 01: Estructura Establecimientos de Hospedaje}

\begin{tabular}{|c|c|c|c|c|c|c|c|}
\hline$\frac{2}{\text { CAIECORIA }}$ & $\begin{array}{c}\mathrm{P} \\
\text { Exalkec } \\
3007\end{array}$ & $\begin{array}{c}\mathrm{N} \\
\text { Establec } \\
.2018 \\
\end{array}$ & $\begin{array}{c}N^{p} \\
\text { Habitac: } \\
2007\end{array}$ & $\begin{array}{c}\text { N } \\
\text { Hahitac: } \\
2008\end{array}$ & $\begin{array}{c}\text { N'Plavas } \\
2007\end{array}$ & $\begin{array}{c}\text { Noftars } \\
2008\end{array}$ & $\begin{array}{l}\text { VAR \% } \\
2007- \\
2003\end{array}$ \\
\hline 1. HOIELS & 25 & 26 & 800 & 820 & 1516 & 1587 & $27 \%$ \\
\hline 3 Extrellas & 6 & 6 & 250 & 250 & 472 & 472 & $0.0 \%$ \\
\hline 2Evatlas & 14 & 15 & 430 & 450 & 812 & 853 & $5.0 \%$ \\
\hline 1 Estella & 5 & 5 & 120 & 120 & 232 & 232 & $0.0 \%$ \\
\hline $21 \% O S T N E S$ & 24 & 28 & 367 & 415 & 714 & 804 & $126 \%$ \\
\hline 3 Estrellis & 3 & 5 & 43 & 71 & 87 & 140 & $609 \%$ \\
\hline 2Estrellas & 14 & 15 & 227 & 236 & 445 & 461 & $3.6 \%$ \\
\hline I Exrella & 7 & 8 & 97 & 108 & 182 & 293 & $11.5 \%$ \\
\hline $\begin{array}{l}\text { 3. No } \\
\text { Catcgoriados }\end{array}$ & 162 & 178 & 2044 & 2267 & 3641 & 4061 & $11.3 \%$ \\
\hline TULAL & 211. & 232 & 3211 & 3E12 & $5 \times 71$ & 6412 & $9.2 \%$ \\
\hline
\end{tabular}


En la categoria general de la oferta de hospedajes los no categorizados resaltan con el $76,72 \%$. (ver Cuadro $\mathrm{N}^{\circ}$ $02)$.

Cuadro $N^{\circ}$ 02: Establecimientos de Hospedaje por Categoria 2008

\begin{tabular}{|c|c|c|}
\hline CATEGORIA & TOTAL & \% \\
\hline HOTELES & 26 & 11,21 \\
\hline HOSTALES & 28 & 12,07 \\
\hline NO CATEG. & 178 & 76,72 \\
\hline TOTAL & $\mathbf{2 3 2}$ & $\mathbf{1 0 0}$ \\
\hline
\end{tabular}

\section{Oferta de Servicios de Bares y Restaurantes}

La investigación consiste en identificar los bares y restaurantes como variables que apoyen al turismo,

El Cuadro $\mathrm{N}^{\circ} 04$ presenta la estructura general de los bares y restaurantes, categorizados y no categorizados existentes en la ciudad de Tacna, Se observa la carencia de restaurantes de mayor calificación de 4 y 5 tenedores, se aprecia la existencia de 1, 2, y 3 tenedores. (Cuadro $\mathrm{N}^{\circ}$ $04)$.

Los bares y restaurantes en el 2008, los categorizados aumentan en 01 y los registrados no categorizados en 23 ; el rubro creció en 24 establecimientos, lo que significa la existencia de pocos restaurantes categorizados, tan solo 13 en relación con el total de 489 del año 2008.(ver Cuadro $\mathrm{N}^{\circ} 05$ ).

Cuadro $N^{\circ} 04$ : Estructura de Restaurantes 2007- 2008

\begin{tabular}{|c|c|c|c|c|c|c|c|}
\hline Categráa & $\begin{array}{c}\text { Nihp } \\
2007\end{array}$ & $\begin{array}{c}\text { N Fipp } \\
208\end{array}$ & $\begin{array}{c}\mathbf{P} \\
\text { Mtsx } \\
2007\end{array}$ & $\begin{array}{c}\mathrm{N} \\
\mathrm{Mks} \\
\mathbf{3} \mathrm{g} 8\end{array}$ & $\begin{array}{c}\text { N SHas } \\
\text { 200? }\end{array}$ & $\begin{array}{c}\text { N STllis } \\
206\end{array}$ & $\begin{array}{l}\text { Ftrsona } \\
\text { Orynd }\end{array}$ \\
\hline Stonodires & 0 & 0 & 0 & 0 & 0 & 0 & 0 \\
\hline 4 tanodins & 0 & 0 & 0 & 0 & 0 & 0 & 0 \\
\hline 3 tonutres & 5 & 6 & III & 121 & 401 & 476 & 37 \\
\hline 2 tenutres & 6 & 6 & 475 & 481 & 1,98 & 2839 & 85 \\
\hline 1 tơức & 1 & 1 & 18 & 18 & 96 & 96 & 8 \\
\hline Rst citegritads & 12 & 13 & 604 & 60 & 292 & 2611 & L30 \\
\hline Regivrads NoGitey & 23 & 246 & 3,438 & 3837 & 14561 & 16199 & 730 \\
\hline NoRegitrads & 230 & $2 \mathbf{2 0}$ & 3806 & 3906 & 1586 & 1586 & 831 \\
\hline TOLNES & +6 & 59 & $78 \times 8$ & 823 & 32918 & 346 & 101 \\
\hline
\end{tabular}

Cuadro N 05 : Oferta de Restaurantes por Categoria $2007-$ 2008

\begin{tabular}{|c|c|c|c|}
\hline Categria & N'Enp 2007 & Y' Enp 2008 & N. Engl Varticion \\
\hline Rest. Categunizados & 12 & 13 & +1 \\
\hline Reyjstrabos No Categ & 223 & 246 & +23 \\
\hline NoRegistrads & 230 & 230 & 0 \\
\hline TOIAIES & 405 & 490 & +24 \\
\hline
\end{tabular}

Podemos señalar que los restaurantes están representados por aquellos registrados y no categorizados en el orden de $50,315 \%$, seguido de los que no están registrados por 47,03 y los clasificados y categorizados solamente significan el $2,66 \%$ del total de 489 en el año 2008. (ver Cuadro $\mathrm{N}^{\circ} 06$ ).

Cuadro N06: Restaurantes por Categoria 2008

\begin{tabular}{|l|c|c|}
\hline \multicolumn{1}{|c|}{ CATEGORIA } & TOTAL & $\%$ \\
\hline Rest. categorizados & 13 & 2,66 \\
\hline Registrados No Categ. & 246 & 50,31 \\
\hline No Registrados & 230 & 47,03 \\
\hline \multicolumn{1}{|c|}{ TOTALES } & $\mathbf{4 8 9}$ & $\mathbf{1 0 0}$ \\
\hline
\end{tabular}

\section{Oferta de Servicios de Agencias de Viajes}

La estructura y la conformación de las agencias de Viajes y Turismo están representadas en el Cuadro $\mathrm{N}^{\circ}$ 09 ; podemos apreciar en este rubro que el $54 \%$ son Minorista, el $42 \%$ son Operadores y las Agencia de Viajes y Turismo Mayorista representa el $4 \%$ del total: (Ver cuadro $\mathrm{N}^{\circ} 09$ ).

Cuadro No 09: Estructura de Agencias de Viajes y Turismo 2007-2008.

\begin{tabular}{|l|c|c|c|c|}
\hline Clasificación & $\begin{array}{c}\mathbf{N}^{\circ} \\
\text { Emp } \\
\mathbf{2 0 0 7}\end{array}$ & $\begin{array}{c}\mathbf{N}^{\circ} \\
\text { Emp } \\
\mathbf{2 0 0 8}\end{array}$ & $\begin{array}{c}\text { VARIACION } \\
\mathbf{N}^{\circ} \text { Emp }\end{array}$ & $\mathbf{2 0 0 8 \%}$ \\
\hline Mayoristas & 1 & 1 & 0 & 4,17 \\
\hline Minoristas & 14 & 13 & -1 & 54,17 \\
\hline Operadoras & 11 & 10 & -1 & 41,67 \\
\hline Total & $\mathbf{2 6}$ & $\mathbf{2 4}$ & -2 & $\mathbf{1 0 0}$ \\
\hline
\end{tabular}

\section{Análisis de la Demanda de Servicios Turísticos}

Las demandas de servicios turísticos deviene de los movimientos migratorios de personas que son los desplazamientos espaciales de grupos sociales, de un lugar a otro,

En el Cuadro $\mathrm{N}^{\circ} 10$ observamos el ingreso de 793,528 chilenos en 2008 sea con cédula de identidad, con salvoconducto y/o con pasaporte. Así mismo, el 2008 ingresaron 727,442 turistas por el terminal Collasuyo.

El índice de pernoctabilidad del turista extranjero es de $78,40 \%$ en relación con el año 2007 y la tendencia es creciente del 2004 al 2007, y el 2008 ha decrecido en $6,9 \%$ en relación a 2007 .

En el Cuadro $\mathrm{N}^{\circ} 11$ se presentan los movimientos migratorios de los visitantes nacionales y los turistas nacionales, ingresaron por el terminal Odría 500,993 personas, por vía aéreo 86,084 , por el terminal Collasuyo ingresaron 250,000 . 


\section{CONCLUSIONES DE LAINVESTIGACIÓN}

1. Se observan en los cuadros y gráficos de análisis que las ofertas turísticas, como son los servicios en establecimientos de hospedajes, los servicios de restaurantes son carentes, frente a una migración importante de extranjeros, especialmente chilenos que arriban permanentemente a la ciudad de Tacna.

2. Según reglamentos, los establecimientos de hospedajes se clasifican de 1 a 5 estrellas, en la ciudad solamente se ofertan hasta tres estrellas, En gran porcentaje existen establecimiento de hospedajes sin clasificación y categorización, con la consecuente atención dificiente o carencia de servicios adecuados para un visitante que exige óptimos servicios en la pernoctación.

3. En general se observa el bajo índice de pernoctabilidad de los turistas y visitantes nacionales y extranjeros y en el ultimo año 2008 la tendencia es negativa, existentes menos arribo de turistas, ya sean nacionales y/o extranjeros.

4. Existe una clasificación de restaurantes, según reglamentos, desde un tenedor hasta cinco tenedores, no existen ningún tipo de restaurantes de 4 y 5 tenedores.

5. En cuanto a la oferta de servicios de las agencias de viajes y turismo, se puede observa en los cuadros de análisis una clara carencia de este tipo de servicios, son servicios que deben y están llamados a fomentar y promocionar el turismo en una ciudad, en este caso de la ciudad y departamento de Tacna, los mismos presentan un variación negativa, es decir, los establecimientos de esta servicio están dejando de operar, los cual tiene una implicancia directa con el desarrollo del turismo departamental, nacional, regional y local.

\section{RECOMENDACIONES}

Como producto del presente trabajo de investigación se recomienda:

\section{En Cuanto se Relaciona al Trabajo de Investigación en General:}

1. Quedan por delante una serie de aspectos por investigar, a fin de completar y profundizar el panorama de la problemática de la oferta y demanda turística de la ciudad y el departamento de Tacna

\section{En Cuanto a los Resultados y Situaciones investigadas:}

2. Referido a los establecimientos de hospedajes debe de fomentarse mayor desarrollo de estas, flexibilizando las gestiones y trámites que para ellos signifiquen engorrosos.

3. El concepto de bar y restaurante podría influir en que los clientes visitantes y turistas demanden y busquen seleccionar un restaurante de mayor categoría en cuanto a la clasificación, es decir de mas tenedores.

En cuanto a la oferta de servicios de los establecimientos de agencias de viajes y turismo, son carentes.

- Disponer en todo momento de una visión conjunta que permita "remar hacia el mismo lado".

- Propiciar la creación de criadero de empresas turísticas en torno a los atractivos turísticos.

- Comprender la importancia de articular una red de políticas conjuntas locales, regionales y nacionales, vinculado a la actividad turística, sin que entren en conflicto.

\section{REFERENCIAS BIBLIOGRÁFICAS}

ACTUALIDAD EMPRESARIAL: Manual del Sistema de Tributación Sectorial, Pacifico Editores, Lima, 2005.

BARBA, Rosa. (1988). "Los nuevos lugares en el territorio. Los espacios del turismo", Revista Geometria, Madrid, 2do semestre.

CLANCY, Michael. (1999). "Turismo y Desarrollo. El caso de México". Annals of Tuorism en Español, Vol.1.

DECRETO SUPREMO No 025-2004- MINCETUR: Restaurantes, noviembre 2005.

DECRETO SUPREMO No 026-2004- MINCETUR: Agencias de Viajes, noviembre 2005.

DECRETO SUPREMO No 029-2004- MINCETUR: Establecimientos de hospedajes, Noviembre 2005

DACHARY, Alfredo César y ARNAIZ BURNE, Stella Maris. (1994.), "Cancún: El enclave turístico y sus costos". Estudios y Perspectivas en Turismo, Vol. 3.

CHEQUER Bauer, Rafael y REJOWSKI, Mirian. (2003). "Oferta turística de Bertioga (SP): caracterizacao, evolucao e análisis", Turismo em Análisis.

SCHLÜTER, Regina.(1994.). "San Carlos de Bariloche: costos y beneficios del ecoturismo". Estudios y Perspectivas en Turismo, Vol. 3,

TOLEDO, Geraldo Luciano, et .al. (2003). "Gestao Interdisciplinar do turismo no planejamento estratégico regional: estudo de casos latino-americanos". Turismo emAnálisis, Vol. 14.

WARD, Barbara. (1976). La morada del hombre. México: Fondo de Cultura Económica.

WILEY. COSHALL, John. Sistema de Turismo (SISTUR) . Estudio del Turismo frente a la Nueva Teoría de los Sistemas. Estudios y Perspectivas en 
Turismo

Brian, Archer. S/f. Demand Forecasting and Estimation. Travel Tourism and Hospitañity Research.: A Handbook for Managers and Researchers, New York, 1993.

Cuadro No 10: Arribo de Turistas y Visitantes Extranjeros a Tacna.
Betech, E. (Julio 2003). "Restaurantes y bares han optado por diferenciarse mediante conceptos novedosos...Van tras los grupos de consumidores sofisticados". A la Carta. Recuperado el 27 de octubre 2004 de:

http://www.soyentrepreneur.com/pagina.hts? $\mathrm{N}=13885$ $\& \mathrm{Ad}=\mathrm{S}$

\begin{tabular}{|c|c|c|c|c|c|c|}
\hline Concepto & 2004 & 2005 & 2006 & 2007 & 2008 & $\begin{array}{l}\text { V. \% } \\
2007- \\
2008\end{array}$ \\
\hline Total arribo Turistas y Visitantes & 574,967 & 700,717 & 870,153 & 917,712 & $1,584,216$ & $72,6 \%$ \\
\hline $\begin{array}{l}\text { 1.- Ingreso chilenos con Cédula de } \\
\text { Identidad }\end{array}$ & 435,792 & 567,609 & 756,202 & 815,368 & 763,873 & $-6,3 \%$ \\
\hline $\begin{array}{lcc}2 .- & \text { Ingreso } & \text { chilenos con } \\
\text { Salvoconducto } & & \\
\end{array}$ & 61,042 & 58,761 & 44,134 & 37,282 & 27,341 & $-26,7 \%$ \\
\hline 3.- Ingreso chilenos con Pasaporte & 0 & 0 & 0 & 0 & 2,314 & $-\because-$ \\
\hline $\begin{array}{l}\text { 4.-TOTAL INGRESO } \\
\text { CHILENOS }(\mathbf{1}+\mathbf{2}+\mathbf{3})\end{array}$ & 496,834 & 626,370 & 800,336 & 852,650 & 793,528 & $-6,9 \%$ \\
\hline $\begin{array}{l}\text { 5.- Chilenos considerados turistas } \\
(28 \% \text { de } 4)\end{array}$ & 139,114 & 175,384 & 224,094 & 238,742 & 222,188 & $-6,9 \%$ \\
\hline $\begin{array}{l}\text { 6.- Chilenos considerados visitantes } \\
\text { - dia }(72 \% \text { de } 4)\end{array}$ & 357,720 & 450.986 & 576,242 & 613,908 & 571,340 & $-6,9 \%$ \\
\hline $\begin{array}{ll}\text { 7.- Ingreso extranjeros con } \\
\text { Pasaporte }\end{array}$ & 78,133 & 74,347 & 69,383 & 65,062 & 63,246 & $-2,8 \%$ \\
\hline $\begin{array}{l}\text { 8.- Ingreso extranjeros Decisión } \\
503 \text { - CAN }\end{array}$ & 0 & 0 & 434 & 0 & 0 & $\because-$ \\
\hline $\begin{array}{l}\text { 9.- Considerados Turistas Terminal } \\
\text { T. Collasuyo }(3 \%)\end{array}$ & s.i. & s.i. & s.i. & s.i. & 727,442 & $\because-$ \\
\hline $\begin{array}{l}\text { 10.- Total chilenos y extranjeros } \\
\text { turistas }(3+5+7+8+9)\end{array}$ & 217,247 & 249,731 & 293,911 & 303,804 & $1,015,190$ & $234,2 \%$ \\
\hline $\begin{array}{l}\text { 11.- Turistas extranjeros que } \\
\text { pernoctan en Tacna }\end{array}$ & 47,741 & 49,646 & 73,853 & 92,438 & 796,094 & $761,2 \%$ \\
\hline $\begin{array}{l}\text { 12.- Porcentaje de turistas que } \\
\text { pernocta en Tacna }\end{array}$ & $22,0^{\circ} \%$ & $19,9 \%$ & $25,1 \%$ & $30,4 \%$ & $78,4 \%$ & $157,7 \%$ \\
\hline $\begin{array}{l}\text { 13.- Indice pernoctabilidad } \\
\text { turista extranjero }\end{array}$ & 1.39 & 1.39 & 1.42 & 1.47 & 1.43 & $-2,7 \%$ \\
\hline $\begin{array}{l}\text { 14.- Gasto percápita diario turista } \\
\text { extranjero (US\$) }\end{array}$ & 55 & 55 & 55 & 55 & 64 & $16,4 \%$ \\
\hline $\begin{array}{l}\text { 15.- Ingresos por turistas } \\
\text { extranjeros }(11 \times 13 \times 14) \text { (US\$) }\end{array}$ & $3,649,799$ & $3,795,437$ & $5,767,919$ & $7,473,612$ & $72,858,508$ & $874,9 \%$ \\
\hline $\begin{array}{l}\text { 16.- Gasto percápita diario visitante } \\
\text { extranjero (US\$) }\end{array}$ & 46 & 46 & 46 & 46 & 55 & $19,6 \%$ \\
\hline $\begin{array}{lll}\text { 17.- Ingresos por visitantes } \\
\text { extranjeros }(6 \times 16) \text { (USS) }\end{array}$ & $\begin{array}{c}16,455,14 \\
2 \\
\end{array}$ & $\begin{array}{c}20,745,37 \\
4 \\
\end{array}$ & $\begin{array}{c}26,507,12 \\
8 \\
\end{array}$ & $\begin{array}{c}28,239,76 \\
8 \\
\end{array}$ & $31,423,709$ & $11,3 \%$ \\
\hline $\begin{array}{l}\text { TOTAL INGRESOS POR } \\
\text { EXTRANJEROS USS }(15+17)\end{array}$ & $20,104,942$ & $24,540,811$ & $32,275,048$ & $35,713,380$ & $104,282,217$ & $192,0 \%$ \\
\hline
\end{tabular}




\begin{tabular}{|c|c|c|c|c|c|c|}
\hline Concepto & 2004 & 2005 & 2006 & 2007 & 2008 & $\begin{array}{c}\text { V. } \% 07- \\
08\end{array}$ \\
\hline $\begin{array}{c}\text { Total arribo Turistas y } \\
\text { Visitantes }(1+2)\end{array}$ & 542,495 & 559,289 & 589,811 & 619,302 & 837,077 & $35,2 \%$ \\
\hline $\begin{array}{l}\text { 1.- Ingreso estimado Terminal } \\
\text { Terrestre M.A. Odría }\end{array}$ & 477,289 & 493,166 & 521,551 & 547,629 & 500,993 & $-8,5 \%$ \\
\hline $\begin{array}{l}\text { 2.- Ingreso estimado Terminal } \\
\text { Aéreo }\end{array}$ & 65,206 & 66,123 & 68,260 & 71,673 & 86,084 & $20,1 \%$ \\
\hline $\begin{array}{l}\text { 3.- Ingreso estimado Terminal } \\
\text { Terrestre Collasuyo }\end{array}$ & s.i. & s.i. & s.i. & s.i. & 250,000 &.-- \\
\hline $\begin{array}{l}\text { 4.- Considerados Turistas } \\
\text { Terminal T. Odría }(37 \%)\end{array}$ & 176,597 & 182,471 & 192,974 & 202,623 & 185,367 & $-8,5 \%$ \\
\hline $\begin{array}{l}\text { 5.- Considerados Turistas } \\
\text { Terminal Aéreo }(10 \%)\end{array}$ & 6,521 & 6,612 & 6,826 & 7,167 & 8,608 & $20,1 \%$ \\
\hline $\begin{array}{l}\text { 6.- Considerados Turistas } \\
\text { Terminal T. Collasuyo ( } 2 \%)\end{array}$ & s.i. & s.i. & s.i. & s.i. & 5,000 &.-- \\
\hline $\begin{array}{l}\text { 7.- Total ingreso Turistas } \\
\text { Nacionales }(4+5+6)\end{array}$ & 183,118 & 189,084 & 199,800 & 209,790 & 198,976 & $-5,2 \%$ \\
\hline $\begin{array}{l}\text { 8.- Turistas Nacionales que } \\
\text { pernoctan en Tacna }\end{array}$ & 264,000 & 274,929 & 295,496 & 328,163 & 259,402 & $-21,0 \%$ \\
\hline $\begin{array}{l}\text { 9.- Porcentaje de Turistas que } \\
\text { pernocta en Tacna }\end{array}$ & $144,2 \%$ & $145,4 \%$ & $147,9 \%$ & $156,4 \%$ & $130,4 \%$ & $-16,7 \%$ \\
\hline $\begin{array}{l}\text { 10.- Índice pernoctabilidad } \\
\text { Turista Nacional }\end{array}$ & 1.39 & 1.39 & 1.42 & 1.37 & 1.33 & $-2,9 \%$ \\
\hline $\begin{array}{l}\text { 11.- Gasto percápita diario } \\
\text { Turista Nacional (US\$) }\end{array}$ & 106 & 106 & 106 & 106 & 148 & $39,6 \%$ \\
\hline $\begin{array}{l}\text { 12.- Ingreso por Turistas } \\
\text { Internos }(8 \times 10 \times 11) \text { (US\$) }\end{array}$ & $38,897,760$ & $40,508,039$ & $44,478,058$ & $47,655,831$ & $51,060,690$ & $7,1 \%$ \\
\hline $\begin{array}{l}\text { 13.- Considerados Visitantes } \\
\text { Nacionales }(40 \% \text { de } 1)\end{array}$ & 190,916 & 197,266 & 208,620 & 219,051 & 200,397 & $-8,5 \%$ \\
\hline $\begin{array}{l}\text { 14.- Gasto percápita diario } \\
\text { Visitante Nacional (US\$) }\end{array}$ & 86 & 86 & 86 & 86 & 121 & $40,7 \%$ \\
\hline $\begin{array}{l}\text { 15.- Ingreso por Visitantes } \\
\text { Nacionales }(11 \times 12) \text { (US\$) }\end{array}$ & $16,418,742$ & $16,964,910$ & $17,941,354$ & $18,838,422$ & $24,248,061$ & $28,7 \%$ \\
\hline $\begin{array}{l}\text { TOTAL INGRESOS POR } \\
\text { NACIONALES USS }(8+11)\end{array}$ & $55,316,502$ & $57,472,949$ & $62,419,412$ & $66,494,253$ & $75,308,751$ & $13,3 \%$ \\
\hline
\end{tabular}

